\title{
Radio and submillimetre observations of wind structure in $\zeta$ Puppis
}

\author{
R. Blomme ${ }^{1}$, G. C. van de Steene ${ }^{1}$, R. K. Prinja ${ }^{2}$, M. C. Runacres ${ }^{1}$, and J. S. Clark ${ }^{2}$ \\ 1 Royal Observatory of Belgium, Ringlaan 3, 1180 Brussel, Belgium \\ 2 Department of Physics \& Astronomy, University College London, Gower Street, London WC1E 6BT, UK
}

Received 17 April 2003 / Accepted 24 June 2003

\begin{abstract}
We present radio and submillimetre observations of the O4I(n)f star $\zeta$ Pup, and discuss structure in the outer region of its wind $\left(\sim 10-100 R_{*}\right)$. The properties of bremsstrahlung, the dominant emission process at these wavelengths, make it sensitive to structure and allow us to study how the amount of structure changes in the wind by comparing the fluxes at different wavelengths. Possible forms of structure at these distances include Corotating Interaction Regions (CIRs), stochastic clumping, a disk or a polar enhancement. As the CIRs are azimuthally asymmetric, they should result in variability at submillimetre or radio wavelengths. To look for this variability, we acquired 3.6 and $6 \mathrm{~cm}$ observations with the Australia Telescope Compact Array (ATCA), covering about two rotational periods of the star. We supplemented these with archive observations from the NRAO Very Large Array (VLA), which cover a much longer time scale. We did not find variability at more than the $\pm 20 \%$ level. The long integration time does allow an accurate determination of the fluxes at 3.6 and $6 \mathrm{~cm}$. Converting these fluxes into a mass loss rate, we find $\dot{M}=3.5 \times 10^{-6} M_{\odot} / \mathrm{yr}$. This value confirms the significant discrepancy with the mass loss rate derived from the $\mathrm{H} \alpha$ profile, making $\zeta$ Pup an exception to the usually good agreement between the $\mathrm{H} \alpha$ and radio mass loss rates. To study the run of structure as a function of distance, we supplemented the ATCA data by observing $\zeta$ Pup at $850 \mu \mathrm{m}$ with the James Clerk Maxwell Telescope (JCMT) and at $20 \mathrm{~cm}$ with the VLA. A smooth wind model shows that the millimetre fluxes are too high compared to the radio fluxes. While recombination of helium in the outer wind cannot be discounted as an explanation, the wealth of evidence for structure strongly suggests this as the explanation for the discrepancy. Model calculations show that the structure needs to be present in the inner $\sim 70 R_{*}$ of the wind, but that it decays significantly, or maybe even disappears, beyond that radius.
\end{abstract}

Key words. stars: early-type - stars: individual: $\zeta$ Pup - stars: mass loss - stars: winds, outflows - radio continuum: stars

\section{Introduction}

$\zeta$ Puppis (HD 66811; O4I(n)f) is one of the most-studied O-type stars. Its stellar wind is driven by radiation pressure, whereby the momentum of the stellar photons is transferred to the wind material via line opacity. With other early-type stars it shares the property that its wind is structured. Evidence for this structure for O-type stars in general is seen in the Discrete Absorption Components (DACs) and black troughs in the ultraviolet resonance lines (e.g. Prinja et al. 1990), the presence of X-ray emission (Sciortino et al. 1990) and the excess flux at infrared and millimetre wavelengths (Runacres \& Blomme 1996). Specifically for $\zeta$ Pup, the significant discrepancy between the mass loss rate derived from the $\mathrm{H} \alpha$ spectral line and that from the radio continuum flux (Petrenz \& Puls 1996) also points to structure in the wind.

Various types of structure have been proposed to explain the observations. Inhomogeneities on the stellar surface (e.g., due to non-radial pulsations), or magnetic fields, result in

Send offprint requests to: $\mathrm{R}$. Blomme,

e-mail: Ronny.Blomme@oma . be somewhat different radiative forces, thereby creating fast and slow streams of gas. As these streams also rotate, they collide, creating Corotating Interaction Regions (CIRs). These are large-scale, spiral-shaped structures in the wind that corotate with the stellar surface. In some cases, the CIRs can explain the most interesting property of DACs: the recurrence time scale is in agreement with the estimated rotation period (Mullan 1986; Cranmer \& Owocki 1996).

Another type of structure that can exist in the wind is of a smaller-scale, stochastic type. It is caused by the inherent instability of the radiative driving mechanism (Owocki 2000). Stochastic structure has been used to explain the X-ray fluxes (Lucy 1982a; Hillier et al. 1993; Feldmeier et al. 1997), the black troughs in the UV resonance lines (Lucy 1982b) and the excess millimetre flux (Blomme et al. 2002).

Finally, it should be noted that $\zeta$ Pup is a rapid rotator (at $43 \%$ of critical velocity). This suggests the possibility that $\zeta$ Pup has a disk, or at least some enhancement of its density near the equatorial plane. Harries \& Howarth (1996) derived a density contrast of at least 1.3 from their linear spectropolarimetry of $\mathrm{H} \alpha$. Such equatorial density enhancement has 
been used (Petrenz \& Puls 1996) to explain the discrepancy that smooth and spherically symmetric models give for the mass loss rates derived from $\mathrm{H} \alpha$ and radio observations.

While observational evidence for the existence of disks in certain stars (e.g. Be stars) is strong, there are theoretical difficulties in understanding how these disks form (Owocki et al. 1998). One of the explanations proposed was the windcompressed disk model (Bjorkman \& Cassinelli 1993), but a more detailed study showed that the non-radial component of the line force and gravity darkening can combine to inhibit the formation of the disk. Interestingly, in some circumstances, the detailed models show an enhancement of the density towards the poles rather than the equator (Owocki et al. 1996, 1998; Petrenz \& Puls 2000).

Observations at submillimetre and radio wavelengths are relevant to structure. This is because bremsstrahlung, the dominant emission process at these wavelengths, has two interesting properties. First, its opacity is proportional to the wavelength squared. Therefore, the observed emission originates above a characteristic radius that increases with wavelength (typical values are $\sim 5 R_{*}$ at $1 \mathrm{~mm}$ and $\sim 100 R_{*}$ at $20 \mathrm{~cm}$ ). Secondly, free-free emission also depends on the density squared. This makes it a good indicator of structure: a structured wind will have more radio emission than a smooth one. By looking for variability at a certain wavelength, one can hope to distinguish between structure that has azimuthal symmetry (stochastic, disk or polar outflow) and structure that lacks azimuthal symmetry (CIRs). By looking at increasing wavelengths, one can see how the "amount" of structure changes in the wind.

We have already applied these techniques to the submillimetre and radio observations of the B0Ia star $\epsilon$ Ori (Blomme et al. 2002). For this star, we did not detect variations in the radio emission (at the $25 \%$ level), but we found that the millimetre flux is substantially higher than a smooth wind model predicts. This discrepancy was interpreted with a model for stochastic structure, showing that considerable structure must persist up to at least $\sim 10 R_{*}$ in the wind of $\epsilon$ Ori. The present paper extends the study of structure in the outer wind to a luminous early O-type star.

To look for variability at radio wavelengths, we acquired 3.6 and $6 \mathrm{~cm}$ observations with the Australia Telescope Compact Array ${ }^{1}$ (ATCA), during a 12-day observing session, which covers about two rotational periods of the star. To see how structure changes as a function of distance, observations were collected at $850 \mu \mathrm{m}$ with the James Clerk Maxwell Telescope ${ }^{2}$ (JCMT), and at $20 \mathrm{~cm}$ with the NRAO Very Large Array $^{3}$ (VLA). We supplemented this material with data from the VLA archive. A log of all data used in this paper is given

\footnotetext{
${ }^{1}$ The Australia Telescope is funded by the Commonwealth of Australia for operation as a National Facility managed by CSIRO.

2 The JCMT is operated by the Joint Astronomy Centre in Hilo, Hawaii on behalf of the parent organizations Particle Physics and Astronomy Research Council in the UK, the National Research Council of Canada and The Netherlands Organization for Scientific Research.

3 The National Radio Astronomy Observatory is a facility of the National Science Foundation operated under cooperative agreement by Associated Universities, Inc.
}

Table 1. New observations and archive data of $\zeta$ Pup.

\begin{tabular}{lll}
\hline \hline $\begin{array}{c}\text { Programme } \\
\text { name }\end{array}$ & Date & $\begin{array}{l}\lambda \\
(\mathrm{cm})\end{array}$ \\
\hline New observations & \\
\hline ATCA & & \\
C824 & $1999-09-15 \rightarrow 27$ & $3.6+6$ \\
VLA & & \\
AB1017 & $2002-03-27$ & 20 \\
JCMT & & \\
M00BU20 & $2000-10-12$ & 0.0850 \\
\hline Archive data & & \\
\hline VLA & & \\
FLOR & $1978-07-23$ & 6 \\
FLOR & $1978-10-13$ & 6 \\
BIEG & $1978-11-05$ & 6 \\
NEWE & $1979-02-09$ & 2 \\
FLOR & $1979-02-16$ & 20 \\
CHUR & $1979-07-12$ & 6 \\
FLOR & $1981-10-18$ & $1.3+2+6+20$ \\
AA28 & $1984-03-07$ & $2+6$ \\
AB327 & $1985-01-29$ & $2+6$ \\
AH365 & $1989-05-13$ & 3.6 \\
AC308 & $1995-01-17$ & 20 \\
\hline
\end{tabular}

in Table 1. Upper limits from other observations (not discussed here) are listed in Wendker (1995).

In Sect. 2, we present the stellar parameters of $\zeta$ Pup. The ATCA, VLA and JCMT observations are discussed in Sects. 3-5 respectively. The interpretation of the observational material is discussed in Sect. 6 and conclusions are drawn in Sect. 7.

\section{The parameters of $\zeta$ Pup}

Table 2 lists the $\zeta$ Pup parameters we use in this paper. The stellar parameters and wind parameters are taken from the unified NLTE model of Puls et al. (1996). These values are in close agreement with models by Bohannan et al. (1990) and Pauldrach et al. (1994). The traditionally assumed distance of 450 pc (Kudritzki et al. 1983) falls well within the Hipparcos error bar, so we will use $d=450$ pc throughout this paper. The radius of $19 R_{\odot}$ at a distance of $450 \mathrm{pc}$ corresponds to an angular diameter of 0.39 milli-arcsec (mas), in good agreement with interferometric measurements $\left(\theta_{\mathrm{LD}}=0.42 \pm 0.03\right.$ mas; Hanbury Brown et al. 1974).

$\zeta$ Pup shows considerable evidence for structure in its stellar wind. Discrete Absorption Components (DACs) are seen to move through the ultraviolet resonance lines (Prinja et al. 1992 and references therein). In 1995, $\zeta$ Pup was one of three targets observed continuously during 16 days by the International Ultraviolet Explorer (IUE Mega Campaign, Massa et al. 1995). The analysis of this high-quality dataset by 
Table 2. Stellar parameters of $\zeta$ Pup.

\begin{tabular}{lll}
\hline \hline Parameter & Value & Reference \\
\hline RA (J2000) & $08^{\mathrm{h}} 03^{\mathrm{m}} 35^{\mathrm{s}} .0467$ & SIMBAD \\
Dec $(\mathrm{J} 2000)$ & $-40^{\circ} 00^{\prime} 11^{\prime \prime} 332$ & catalogue \\
$\mu_{\alpha} \cos \delta$ & $-31.7 \pm 0.5 \mathrm{mas} / \mathrm{yr}$ & $\mathrm{G} 01$ \\
$\mu_{\delta}$ & $+17.6 \pm 0.6 \mathrm{mas} / \mathrm{yr}$ & $\mathrm{G} 01$ \\
$V$ magnitude & 2.25 & $\mathrm{M} 87$ \\
$B-V$ & -0.27 & $\mathrm{M} 87$ \\
$E_{B-V}$ & 0.044 & $\mathrm{~S} 77$ \\
Spectral type & $\mathrm{O} 4 \mathrm{I}(\mathrm{n}) \mathrm{f}$ & $\mathrm{W} 72$ \\
$T_{\text {eff }}$ & $42000 \mathrm{~K}$ & $\mathrm{P} 96$ \\
$\log g$ & 3.60 & $\mathrm{P} 96$ \\
$\log L / L_{\odot}$ & 6.00 & $\mathrm{P} 96$ \\
$R_{*}$ & $19 R_{\odot}$ & $\mathrm{P} 96$ \\
$M_{*}$ & $52.5 M_{\odot}$ & $\mathrm{P} 96$ \\
$N_{\mathrm{He}} / N_{\mathrm{H}}$ & 0.12 & $\mathrm{P} 96$ \\
$v \sin i$ & $220 \mathrm{~km} \mathrm{~s}^{-1}$ & $\mathrm{P} 96$ \\
$v_{\infty}$ & $2250 \mathrm{~km} \mathrm{~s}^{-1}$ & $\mathrm{P} 96$ \\
$\beta$ & 1.15 & $\mathrm{P} 96$ \\
$\dot{M}$ & $5.9 \times 10^{-6} M_{\odot} / \mathrm{yr}$ & $\mathrm{P} 96$, from H$\alpha$ \\
$d$ & $450 \mathrm{pc}^{+120} \mathrm{pc}$ & $\mathrm{K} 83$ \\
& $429_{-77}$ & Hipparcos \\
\hline
\end{tabular}

References: G01 Gontcharov et al. (2001), K83 Kudritzki et al. (1983), M87 average from Mermilliod (1987), P96 Puls et al. (1996), S77 Snow et al. (1977), W72 Walborn (1972).

Howarth et al. (1995) reveals a $19.2 \mathrm{~h}$ and a 5.2 day period. The $19.2 \mathrm{~h}$ period is the recurrence time of the DACs. The 5.2 day period indicates modulation on a global scale, and is in good agreement with the rotation period estimated from $v \sin i$, suggesting that material in the wind corotates with the star. One possible cause for this corotating material is the presence of a (weak) magnetic field. Attempts to detect the magnetic field for $\zeta$ Pup have so far yielded a null result with an uncertainty of 100-200 G (Barker et al. 1981; Chesneau \& Moffat 2002).

$\zeta$ Pup also shows variability in the $\mathrm{H} \alpha$ and He II 4686 line profiles (Moffat \& Michaud 1981 and references therein, Hendry \& Bahng 1981; Reid \& Howarth 1996; Eversberg et al. 1998). The presence of variability in these wind-formed lines is consistent with large-scale structures corotating through the wind. Eversberg et al. however interpret their He II 4686 observations in terms of smaller-scale structures ("clumps") moving out in the wind. Fullerton et al. (1996) and Reid \& Howarth (1996) detected variability in a number of optical lines (He I, He II, N IV, C IV). The variations have a period of $8.54 \mathrm{~h}$. Both papers attempt to interpret the variations in terms of non-radial pulsations, but they also raise concerns that these lines might be wind contaminated. The photospheric perturbations due to nonradial pulsations are another possible way of creating CIRs.
Optical continuum fluxes also show variability: the 5.2 day rotation period was detected in optical photometry (Balona 1992). From Hipparcos photometry, Marchenko et al. (1998) derive a period which is half the rotation period.

Other indicators of structure are the continuum flux excess at infrared and millimetre wavelengths (Runacres \& Blomme 1996) and the presence of X-ray emission (Long \& White 1980). Models by Hillier et al. (1993) and Feldmeier et al. (1997) show that structure can explain the X-ray emission. Earlier claims that the observed X-ray emission is variable (Collura et al. 1989) were later shown to be incorrect (Berghöfer \& Schmitt 1994). Later, Berghöfer et al. (1994) found variability in the ROSAT X-ray data (on roughly the same time scale as the $\mathrm{H} \alpha$ variability), but this was not confirmed by the ASCA data (Oskinova et al. 2001).

\section{ATCA observations}

\subsection{Data}

We used the Australia Telescope Compact Array (ATCA) for a long observing session on $\zeta$ Pup in September 1999. The $\sim 27 \mathrm{~h}$ on-target integration time was divided into 8 runs, spread over 12 days, thus providing coverage of the $\sim 5.2$ day rotation period. A detailed observing log is presented in Table 3. At the time of the observation, ATCA was in configuration 6A, with the longest baseline at $5939 \mathrm{~m}$ and the shortest one at $337 \mathrm{~m}$. The continuum observations were done simultaneously at $3.6 \mathrm{~cm}(X$-band; $8.688 \mathrm{GHz})$ and $6 \mathrm{~cm}(C$-band; $4.848 \mathrm{GHz}$ ). The observing bandwidth was $128 \mathrm{MHz}$. A single observing run consists of repetitively observing $\zeta$ Pup for $15 \mathrm{~min}$ and then the phase calibrator PKS B0823-500 for $5 \mathrm{~min}$. The flux calibrator PKS B1934-638 was observed when possible at the beginning or end of the run. The flux calibrator could not be observed during the fourth (SEP20) and seventh observing run (SEP25) due to scheduling or technical problems.

\subsection{Reduction}

As the level of variability we expect is small, the reduction needs to be done as carefully as possible. For this reason, we also discuss the reduction in detail. The data were reduced in Miriad following the user guide (Sault \& Killeen 1999). The data were read into Miriad and corrected for self-interference of the array, as well as for the phase difference between the $X$ and $Y$ channels. Next, the programme BLFLAG was used to flag out bad datapoints interactively. The calibrators were then used to determine the antenna gains as a function of time (using MFCAL).

The flux assigned to the flux calibrator PKS B1934-638 is $5.85 \mathrm{Jy}$ (with a $2 \%$ error) at $6 \mathrm{~cm}$. The flux value determined with UVFLUX of the phase calibrator PKS B0823-500 is $3.0 \mathrm{Jy}$. At $3.6 \mathrm{~cm}$ the flux calibrator is $2.88 \mathrm{Jy}$ (with a $2 \%$ error) and the phase calibrator is $1.53 \mathrm{Jy}$. In those runs where the flux calibrator had not been observed, we used the phase calibrator with the above mentioned flux values to calibrate the data. We determined the bandpass function from the flux calibrator. 
Table 3. ATCA data for $\zeta$ Pup. Start and end times of each observing run are given, as well as the total duration of the run and the integration time on $\zeta$ Pup. The measured fluxes at 3.6 and $6 \mathrm{~cm}$ are listed, with their error bars and the size of the beams (major $\times$ minor axis and position angle). The data obtained on SEP23-24 were split in two sets in order to have the same time span as in the other observing runs.

\begin{tabular}{|c|c|c|c|c|c|c|c|c|}
\hline $\begin{array}{c}\text { Start date-time } \\
1999-\end{array}$ & $\begin{array}{c}\text { End date-time } \\
\text { 1999- }\end{array}$ & $\begin{array}{l}\text { Total } \\
\text { time (h) }\end{array}$ & $\begin{array}{l}\zeta \text { Pup } \\
\text { time (h) }\end{array}$ & $\begin{array}{l}F_{3.6 \mathrm{~cm}} \\
(\mathrm{mJy})\end{array}$ & $\begin{array}{l}F_{6 \mathrm{~cm}} \\
(\mathrm{mJy})\end{array}$ & $\begin{array}{c}\text { Beam }_{3.6 \mathrm{~cm}} \\
\left({ }^{\prime \prime} \times{ }^{\prime \prime}\right)\end{array}$ & $\begin{array}{c}\text { PA } \\
(\operatorname{deg})\end{array}$ & $\begin{array}{c}\text { Beam }_{6 \mathrm{~cm}} \\
\left({ }^{\prime \prime} \times{ }^{\prime \prime}\right)\end{array}$ \\
\hline SEP15-23:47:55 & SEP16-03:32:15 & 3.30 & 2.44 & $1.98 \pm 0.12$ & $1.40 \pm 0.11$ & $4.05 \times 0.68$ & 33.6 & $7.43 \times 1.23$ \\
\hline SEP18-23:07:15 & SEP19-03:01:15 & 3.52 & 2.56 & $2.10 \pm 0.12$ & $1.49 \pm 0.11$ & $3.78 \times 0.67$ & 29.4 & $6.96 \times 1.20$ \\
\hline SEP19-22:09:05 & SEP20-01:59:35 & 3.44 & 2.50 & $1.98 \pm 0.13$ & $1.34 \pm 0.11$ & $4.31 \times 0.72$ & 18.3 & $8.07 \times 1.10$ \\
\hline SEP20-18:06:25 & SEP20-22:13:35 & 3.79 & 2.92 & $2.40 \pm 0.12$ & $1.61 \pm 0.11$ & $3.58 \times 0.63$ & -20.4 & $6.63 \times 1.14$ \\
\hline SEP22-20:08:55 & SEP23-02:16:45 & 5.26 & 3.93 & $2.22 \pm 0.13$ & $1.62 \pm 0.10$ & $2.65 \times 0.62$ & 9.5 & $4.82 \times 1.14$ \\
\hline \multirow[t]{2}{*}{ SEP23-15:07:55 } & SEP24-01:38:55 & 9.39 & 6.86 & $2.41 \pm 0.13$ & $1.67 \pm 0.11$ & $2.41 \times 0.79$ & -45.1 & $4.35 \times 1.44$ \\
\hline & & & & $2.40 \pm 0.13$ & $1.66 \pm 0.10$ & $3.22 \times 0.60$ & 9.2 & $5.92 \times 1.09$ \\
\hline SEP25-20:05:25 & SEP26-00:00:25 & 3.54 & 2.74 & $2.55 \pm 0.13$ & $1.60 \pm 0.11$ & $4.11 \times 0.57$ & 2.6 & $7.74 \times 1.04$ \\
\hline SEP27-16:04:05 & SEP27-19:59:35 & 3.60 & 2.62 & $2.39 \pm 0.14$ & $1.53 \pm 0.11$ & $3.30 \times 0.73$ & -40.3 & $6.02 \times 1.32$ \\
\hline \multirow[t]{4}{*}{ TOTAL } & & 35.84 & 26.57 & & & & & \\
\hline & & \multicolumn{2}{|c|}{ average flux } & 2.26 & 1.55 & & & \\
\hline & & \multicolumn{2}{|c|}{ median flux } & 2.39 & 1.60 & & & \\
\hline & & \multicolumn{2}{|c|}{ Complete data set } & $2.38 \pm 0.09$ & $1.64 \pm 0.07$ & $1.65 \times 0.73$ & 2.6 & $3.01 \times 1.33$ \\
\hline
\end{tabular}

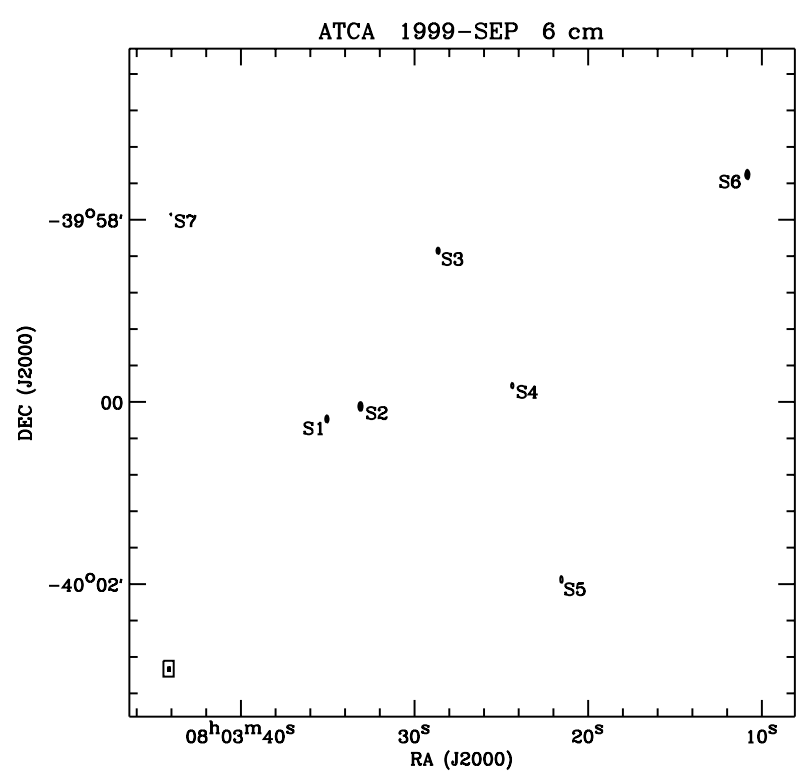

Fig. 1. $\zeta$ Pup and 6 other sources detected at $6 \mathrm{~cm}$ on the ATCA images. This map covers a large part of the primary beam. The synthesized beam is shown in the lower left corner. Identifications of the sources are listed in Table 4. Sources S6 and S7 are not seen on the $3.6 \mathrm{~cm}$ image, because of the smaller primary beam.

We interpolated the instrumental gains and applied them to the $\zeta$ Pup observation. The task INVERT was then used to produce an image from the visibility datasets by Fourier transform. In the inversion we used multi-frequency synthesis (MFS), which compensates for the spectral index of the source across the bandwidth. Because of the sparse UV-coverage per observation, we used robust uniform weighting (Briggs 1995) to improve the rms in the map. To de-convolve the image we used MFCLEAN. The resulting clean components are then
Table 4. Position of other sources on the combined ATCA image. Formal error bars are better than 0.01 in right ascension and $0 \prime^{\prime} 1$ in declination. Identifications given refer to Jones (1985) and Condon et al. (1998). No identification was found for S4 or S7 in the SIMBAD or HEASARC catalogues.

\begin{tabular}{llll}
\hline \hline No. & RA (2000) & Dec (J2000) & Identification \\
\hline S2 & 080333.11 & -400003.1 & EQ 0801-398 \\
S3 & 080328.65 & -395820.4 & NVSS J080327-395828 \\
S4 & 080324.38 & -395949.2 & \\
S5 & 080321.54 & -400156.9 & NVSS J080321-400153 \\
S6 & 080310.87 & -395729.8 & NVSS J080310-395729 \\
S7 & 080344.03 & -395756.5 & \\
\hline
\end{tabular}

convolved with a Gaussian and added to the residual image. Besides $\zeta$ Pup, 6 other objects were detected in the primary beam at $6 \mathrm{~cm}$ and 4 at $3.6 \mathrm{~cm}$ (see Fig. 1 and Table 4).

The cleaning was stopped when the absolute maximum in the residual map (i.e. the map from which the clean components have been subtracted) reached $0.30 \mathrm{mJy}$. This is about 3 to 4 times the theoretical noise of each map, where the theoretical noise is calculated taking only the system temperature of the front-end receiver into account, not the calibration errors, side-lobes or any other instrumental effects. Experience shows that at this cutoff the rms in the cleaned map and in the residual map are both about equal to the theoretical rms. At the end, to correct for primary beam attenuation, the task LINMOS was used.

All images were reduced in exactly the same way with exactly the same parameters. 

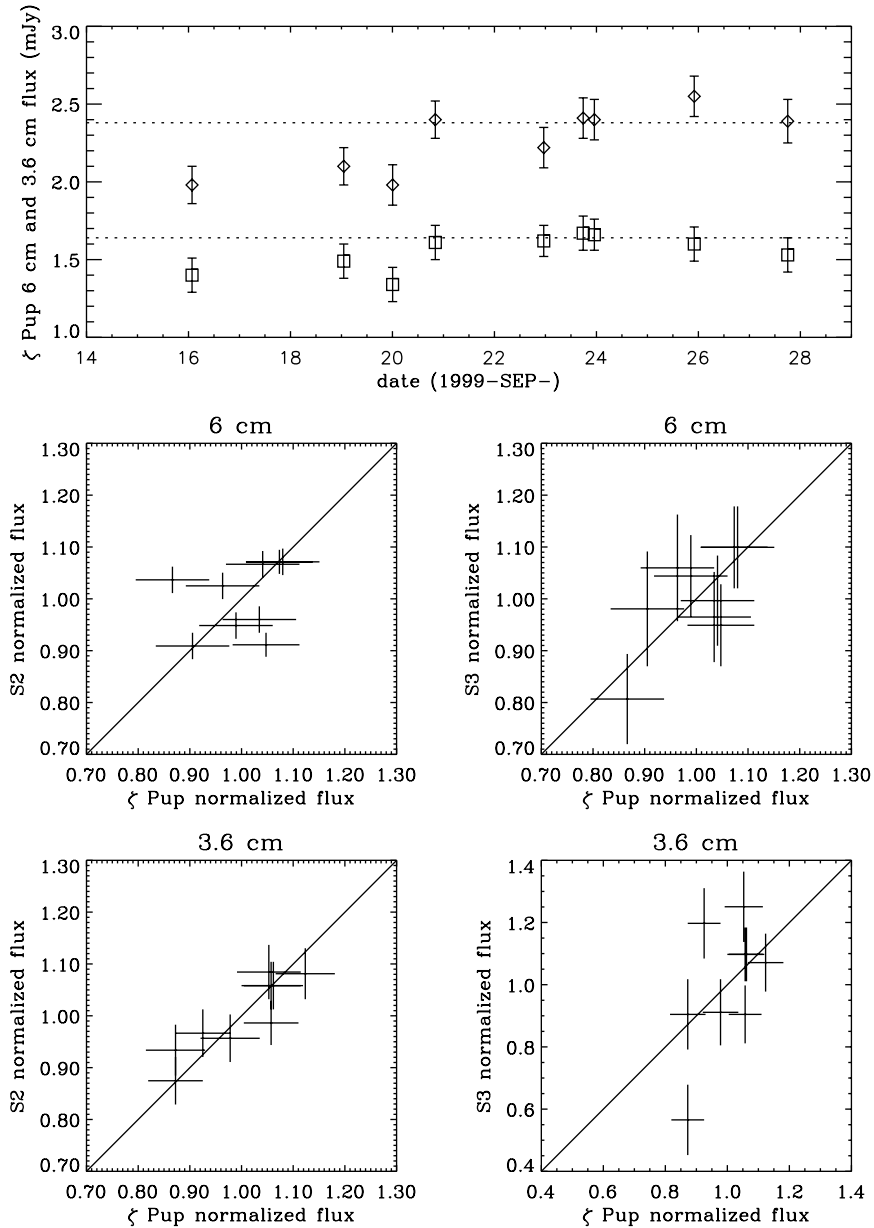

Fig. 2. The top panel shows the $\zeta$ Pup fluxes of each ATCA observation at $3.6 \mathrm{~cm}(\diamond)$ and $6 \mathrm{~cm}(\square)$ as a function of time. The flux derived from the single dataset combining all the ATCA observations is given by the dotted line. The lower 4 panels show the correlation of the $\zeta$ Pup fluxes with those of S2 = EQ 0801-398 and S3 = NVSS J080327395828. In the correlation plots the fluxes were normalized by their average.

\subsection{Fluxes and error bars}

The flux of $\zeta$ Pup was determined by fitting an elliptical Gaussian, with values for the major and minor axis and position angle kept fixed to the beam values. Table 3 lists the flux values at 3.6 and $6 \mathrm{~cm}$, and the respective beam sizes with their position angle. Because the observations at 3.6 and $6 \mathrm{~cm}$ were done simultaneously, the position angles are the same at both wavelengths. The data obtained on SEP23-24 were split in two sets in order to have the same time span as in the other observing runs. The $6 \mathrm{~cm}$ fluxes of the other sources in the image are less than $1.2 \mathrm{mJy}$, except S2 and S6 which are about $4 \mathrm{mJy}$ (uncorrected for the primary beam effect): these values are low enough that we do not have to worry about the effect of their sidelobes. The fitting procedure (IMFIT) gives an rms error on the flux measurement. To take into account the calibration error, we added $2 \%$ of the flux values to this rms, and thus arrived at the final error bars (listed in Table 3).

This error bar only covers the random sources of error. To get a feeling for the systematic errors, we redid the reduction in a slightly different way. Instead of robust uniform weighting, we tried both uniform weighting (by setting the robust parameter to -2 ) and natural weighting (robust parameter $=+2$ ). In the latter, the rms in the maps is lower at the expense of the more elongated beam and worse side-lobe levels. In the former, the beam shape is smaller at the expense of higher rms levels in the map. The robust uniform and natural weighting flux determinations agree well within the error bar. As usual, the better the UV coverage and beam shape the less difference there is among the different flux determinations. We also measured the flux by determining the maximum intensity of the point source, instead of fitting an elliptical Gaussian. This alternative flux determination always falls within the error bar.

\subsection{Results}

The resulting 3.6 and $6 \mathrm{~cm}$ fluxes are plotted in the top panel of Fig. 2. While this figure suggests that $\zeta$ Pup is variable at both wavelengths, we found a similar behaviour in other sources on the map. We therefore compared the flux values for $\zeta$ Pup with two of the brightest sources close-by (S2 = EQ 0801-398 and S3 = NVSS J080327-395828, see Fig. 1). The comparison (lower panels in Fig. 2) shows that all three sources follow the same trend. Hence, this argues strongly against $\zeta$ Pup showing variability. A further argument against variability follows from the fluxes derived from the complete data set (see below) which are also plotted in Fig. 2 (dotted lines). Obviously, the flux of the complete data set is not the straight average of the separate fluxes. This points to problems in the interpolation of the instrumental gain phases during the first few runs of the observing session, causing part of the $\zeta$ Pup flux to be scattered over the rest of the image. These runs get less weight in the complete data set due to the non-linear nature of the cleaning process. Having concluded that the present data do not show detectable variability in $\zeta$ Pup, we can derive upper limits on the amount of variability from the range in fluxes (with their error bars): these are $\pm 20 \%$ of its flux value at 3.6 and at $6 \mathrm{~cm}$.

In principle, the non-detection of variability could be caused by a bad coverage of the phase for the various periods. However, the 8 observations spread over 12 days cover the 5.2 day rotation period reasonably well. We also checked the phase coverage of the variability cycle for possible periods around $8.5 \mathrm{~h}$ and $19 \mathrm{~h}$ (see Sect. 2) and found it to be good.

As there is no variability, we combined all visibility data and Fourier transformed them to obtain a single map. Because the rms in this combined map is lower than in each individual map, we cleaned it deeper, down to $0.1 \mathrm{mJy}$. The resulting images at 3.6 and $6 \mathrm{~cm}$ are shown in Fig. 3. The measured fluxes and their error bars (rms $+2 \%$ of the flux) are listed in Table 3 .

To get a feeling for the systematic errors on the combined data set, we investigated the effect of the cutoff limit in the CLEANing procedure: we CLEANed the total map at $6 \mathrm{~cm}$ (robust $=0.5, \mathrm{rms}=0.024 \mathrm{mJy}$ ) down to $0.07 \mathrm{mJy}$ and $0.15 \mathrm{mJy}$. In the former case the number of clean components was 2769 in the latter 160 . The resulting $\zeta$ Pup fluxes fall well within the error bar. Also using a single, large, cleaning box instead of multiple cleaning boxes gave negligible 

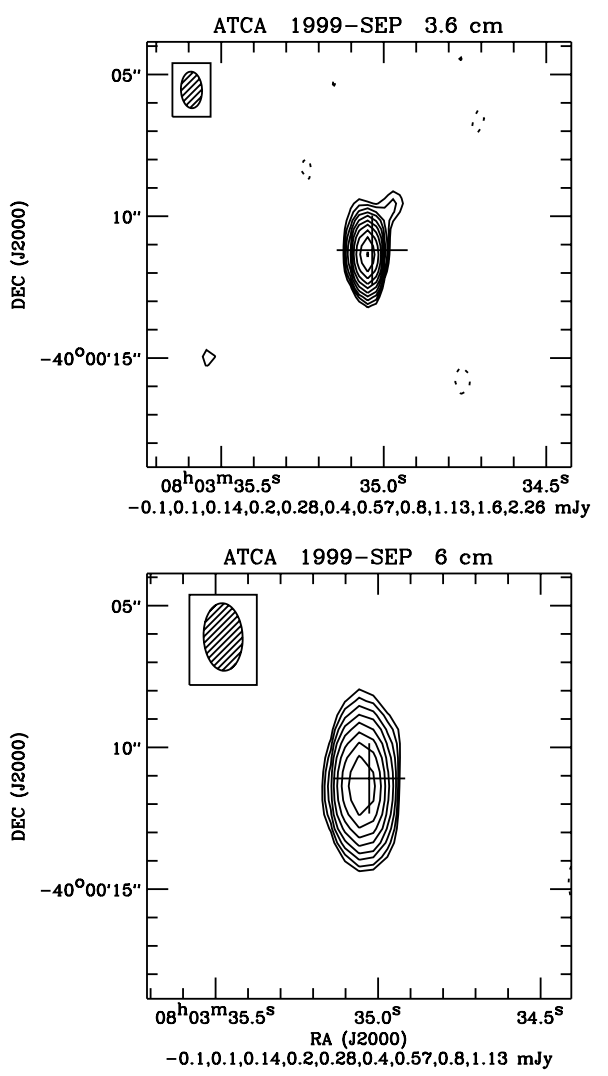

Fig. 3. ATCA observation (all data combined) of $\zeta$ Pup at 3.6 and $6 \mathrm{~cm}$. The cross indicates the optical ICRS 2000.0 position (from SIMBAD), corrected for proper motion (see Table 2). The contour levels follow a logarithmic scale: their values are listed at the bottom of each figure. The negative contour is given by the dashed line. The first positive contour is at about three times the rms noise in the map. The beam is shown in the upper left corner.

differences in the flux. Finally, we redid the reduction, systematically dropping one of the observing runs. The range of values thus obtained again falls within the error bar.

\section{VLA observations}

\section{1. $20 \mathrm{~cm}$ observation}

We obtained a $20 \mathrm{~cm}$ ( $L$-band) observation on 2002 March 27 with the VLA in A configuration (i.e. the configuration with the highest spatial resolution). The observation alternated between $\zeta$ Pup and the phase calibrator 0814-356 (J2000). Two runs of 7 min were made on $\zeta$ Pup and 3 runs of 2 min on the phase calibrator. For the flux calibration we also observed $3 \mathrm{C} 48=0137+331(\mathrm{~J} 2000)$. The observation consists of two sidebands (at 1.3851 and $1.4649 \mathrm{GHz}$ ), each of $50 \mathrm{MHz}$ bandwidth.

The reduction of these data was done using the NRAO package AIPS (Astronomical Image Processing System), following the same steps as for the ATCA data (Sect. 3). Technical details of the reduction are listed in Table 5. We stopped cleaning when the algorithm started finding about the same number of negative as positive components. The resulting map is shown in Fig. 4.

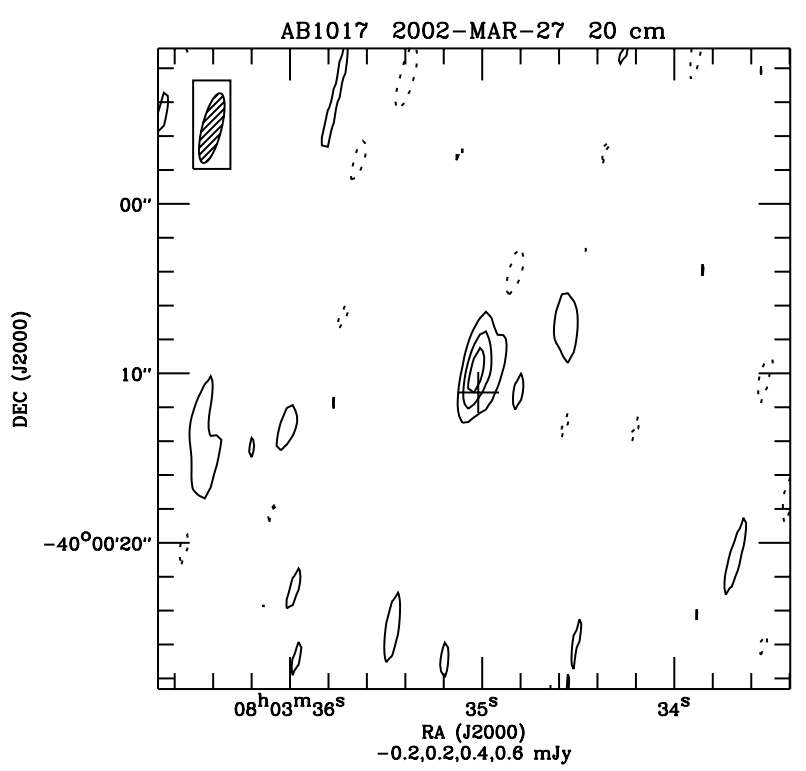

Fig. 4. VLA observation at $20 \mathrm{~cm}$. The contour levels follow a linear scale and the values are listed at the bottom of the figure. The negative contour is given by the dashed line. The first positive contour is at about twice the rms noise in the map. The beam is shown in the upper left corner. The cross indicates the optical ICRS 2000.0 position (from SIMBAD), corrected for proper motion. The $\sim 1$ ". 4 offset with the radio position is most probably due to ionospheric refraction, which is more important at longer wavelengths and at the low elevation at which $\zeta$ Pup was observed.

By fitting an elliptical Gaussian to $\zeta$ Pup, we found a $20 \mathrm{~cm}$ flux of $0.76 \pm 0.09 \mathrm{mJy}$. The error bar is the rms noise in the total map. We also added a $2 \%$ calibration error to the result (Perley \& Taylor 2002), but this does not change the resulting error bar significantly. The maximum intensity of $\zeta$ Pup is $0.74 \mathrm{mJy} /$ beam: the close agreement with the value from the Gaussian fit shows that $\zeta$ Pup is indeed a point source.

To judge the robustness of our flux determination, we repeated the reduction, systematically dropping one antenna, using different weightings of distant visibilities, or natural weighting instead of robust uniform, or doubling or halving the number of clean components. In all cases, the results fall within the error bar. The rms error bar therefore not only covers the statistical uncertainty, but the systematic errors as well.

\subsection{Archive observations}

The VLA archive contains a number of $\zeta$ Pup observations, which are listed in Table 1. Many of these observations have not been published previously. To avoid introducing systematic effects we decided to reduce the whole set. The procedure is the same as that followed in Sect. 4.1. The details of the reductions are summarized in Table 5 . In principle, the $1.3 \mathrm{~cm}$ ( $K$-band) and $2 \mathrm{~cm}$ ( $U$-band) observations should be corrected for differential atmospheric extinction (using the AIPS task ELINT), but in none of those cases was there sufficient information to apply this.

The resulting maps are shown in Fig. 5 and the fluxes are listed in Table 5. The error bars show the range of results found 
Table 5. Reduction of the VLA data. Column 1 gives the wavelength, 2 the programme name, 3 the date of the observation, 4 the configuration the VLA was in at the time of the observation, 5 the name of the flux calibrator used, 6 the flux (in Jy) assigned to the flux calibrator (two values are given if there are two sidebands), 7 the phase calibrator (J2000 coordinates), 8 the number of antennas that gave a usable signal, 9 the integration time (in minutes) on the target, 10 the beamsize (major $\times$ minor axis) in $\operatorname{arcsec}^{2}, 11$ the measured flux (in mJy) and 12 refers to the notes. The fluxes of the flux calibrators are based on Perley \& Taylor (2002).

\begin{tabular}{|c|c|c|c|c|c|c|c|c|c|c|c|}
\hline \multirow{3}{*}{$\begin{array}{c}(1) \\
\lambda \\
(\mathrm{cm})\end{array}$} & \multirow{3}{*}{$\begin{array}{c}(2) \\
\text { Progr. }\end{array}$} & \multirow{3}{*}{$\begin{array}{l}\text { (3) } \\
\text { Date }\end{array}$} & \multirow{3}{*}{$\begin{array}{l}\text { (4) } \\
\text { Conf. }\end{array}$} & (5) & (6) & \multirow{3}{*}{$\begin{array}{c}(7) \\
\text { Phase } \\
\text { calibrator }\end{array}$} & \multirow{3}{*}{$\begin{array}{l}(8) \\
\text { No. } \\
\text { ants. }\end{array}$} & \multirow{3}{*}{$\begin{array}{c}(9) \\
\text { Intgr. } \\
\text { time }\end{array}$} & \multirow{3}{*}{$\begin{array}{c}(10) \\
\text { Beamsize } \\
\left(\operatorname{arcsec}^{2}\right)\end{array}$} & \multirow{3}{*}{$\begin{array}{c}(11) \\
\text { Flux } \\
(\mathrm{mJy})\end{array}$} & \multirow{3}{*}{$\begin{array}{c}(12) \\
\text { Notes }\end{array}$} \\
\hline & & & & \multicolumn{2}{|c|}{ Flux calibrator } & & & & & & \\
\hline & & & & Name & Flux (Jy) & & & & & & \\
\hline 1.3 & FLOR & 1981-10-18 & $\mathrm{C}$ & $3 \mathrm{C} 286$ & 2.517 & $0828-375$ & 19 & 56 & $5.6 \times 3.0$ & $<13$ & 1,2 \\
\hline \multirow[t]{4}{*}{2} & NEWE & 1979-02-09 & & - & - & $0730-116$ & 8 & 9 & - & $<13$ & $1,2,3$ \\
\hline & FLOR & 1981-10-18 & $\mathrm{C}$ & $3 \mathrm{C} 286$ & 3.452 & $0828-375$ & 24 & 64 & $5.1 \times 3.5$ & $4.3 \pm 0.9$ & 1 \\
\hline & AA28 & 1984-03-07 & $\mathrm{CnB}$ & $3 \mathrm{C} 286$ & $3.423 / 3.432$ & $0828-375$ & 24 & 18 & $1.7 \times 1.3$ & $2.9 \pm 0.3$ & 1,4 \\
\hline & AB327 & $1985-01-29$ & A & $3 \mathrm{C} 48$ & $1.742 / 1.748$ & $0828-375$ & 26 & 121 & $3.0 \times 1.8$ & - & $1,5,6$ \\
\hline 3.6 & AH365 & 1989-05-13 & $\mathrm{CnB}$ & $3 \mathrm{C} 48$ & $3.171 / 3.153$ & $0828-375$ & 26 & 14 & $4.0 \times 3.0$ & $1.4 \pm 0.3$ & 6,7 \\
\hline \multirow[t]{7}{*}{6} & FLOR & $1978-07-23$ & & $3 C 286$ & 7.462 & 0836-202 & 11 & 9 & - & $<4$ & 8 \\
\hline & FLOR & 1978-10-13 & & $3 C 286$ & 7.462 & 0836-202 & 9 & 160 & $9.4 \times 1.3$ & $1.7 \pm 0.3$ & 9 \\
\hline & BIEG & 1978-11-05 & & $3 C 286$ & 7.462 & 0836-202 & 8 & 24 & 16. $\times 0.69$ & $<4$ & 8 \\
\hline & CHUR & 1979-07-12 & & $3 \mathrm{C} 286$ & 7.462 & $0828-375$ & 12 & 140 & $4.9 \times 0.55$ & $1.4 \pm 0.3$ & 10,11 \\
\hline & FLOR & 1981-10-18 & $\mathrm{C}$ & $3 \mathrm{C} 286$ & 7.462 & $0828-375$ & 27 & 28 & 18. $\times 3.6$ & $1.71 \pm 0.14$ & \\
\hline & AA28 & 1984-03-07 & $\mathrm{CnB}$ & $3 \mathrm{C} 286$ & $7.462 / 7.510$ & $0828-375$ & 24 & 18 & $5.0 \times 4.1$ & $1.49 \pm 0.11$ & 12 \\
\hline & AB327 & 1985-01-29 & A & $3 \mathrm{C} 48$ & $5.405 / 5.459$ & $0828-375$ & 27 & 91 & $1.1 \times 0.45$ & $1.05 \pm 0.08$ & 6 \\
\hline \multirow[t]{3}{*}{20} & FLOR & $1979-02-16$ & & $3 \mathrm{C} 286$ & 14.51 & 0836-202 & 11 & 182 & 14. $\times 2.3$ & $<1.5$ & 2 \\
\hline & FLOR & 1981-10-18 & $\mathrm{C}$ & $3 C 286$ & 14.51 & $0828-375$ & 26 & 18 & 60. $\times 12$ & $<0.75$ & 2 \\
\hline & AB 1017 & $2002-03-27$ & A & $3 \mathrm{C} 48$ & $15.49 / 16.20$ & 0814-356 & 26 & 14 & $5.5 \times 1.1$ & $0.76 \pm 0.09$ & \\
\hline
\end{tabular}

Notes:

(1) Insufficient data for atmospheric extinction correction.

(2) Upper limit from 3 sigma.

(3) No flux calibrator, used 0532+075 (J2000) instead with an assumed flux of $2.20 \mathrm{Jy}$.

(4) Bieging et al. (1989) list $3.0 \pm 0.2$ mJy for this observation.

(5) Use of 3C 48 as a flux calibrator in the A configuration is not recommended (Perley \& Taylor 2002).

(6) Data reduction problems detailed in Sect. 4.2.

(7) Lamers \& Leitherer (1993) list $1.60 \pm 0.07 \mathrm{mJy}$ for this observation, based on work by Howarth \& Brown (1991).

(8) Upper limit derived from non-detection of EQ 0801-398.

(9) Primary is resolved; true flux might be slightly lower.

(10) An additional $10 \%$ uncertainty in the flux calibration should be added, because the instrumental gains of the flux and phase calibrator show significant differences (Fomalont \& Perley 1999).

(11) Abbott et al. (1980) and Bieging et al. (1989) list $1.4 \pm 0.3 \mathrm{mJy}$ for this observation.

(12) Bieging et al. (1989) list $1.3 \pm 0.1 \mathrm{mJy}$ for this observation.

by various reductions where we systematically dropped one antenna, used different weightings of distant visibilities, or natural weighting instead of robust uniform. The error bars are always larger than the rms noise in the map. For each observation, we compared the peak intensity of the source to the flux derived from the Gaussian fit. With the only exception of the $3.6 \mathrm{~cm}$ observation of AH365 (see below), there is very good agreement, showing that the sources are indeed point sources. When we doubled or halved the number of clean components, we were always well within the error bar. The error bars of the 3.6, 6 and $20 \mathrm{~cm}$ observations include a $2 \%$ calibration error, and the $2 \mathrm{~cm}$ a $5 \%$ calibration error.

As $\zeta$ Pup is rather low on the horizon from the VLA, the quality of the observations is not always good. The AB327 observations at 2 and $6 \mathrm{~cm}$ show large variations in the gain phases (30-50 degrees) of the calibrators. All sources on the $6 \mathrm{~cm}$ image (including $\zeta$ Pup) are 30-50\% lower in flux compared to other observations. The $2 \mathrm{~cm} \zeta$ Pup flux is heavily dependent on tapering, and is compatible with a value of $\sim 3 \mathrm{mJy}$. The $3.6 \mathrm{~cm}$ observation of AH365 is the only one where the 

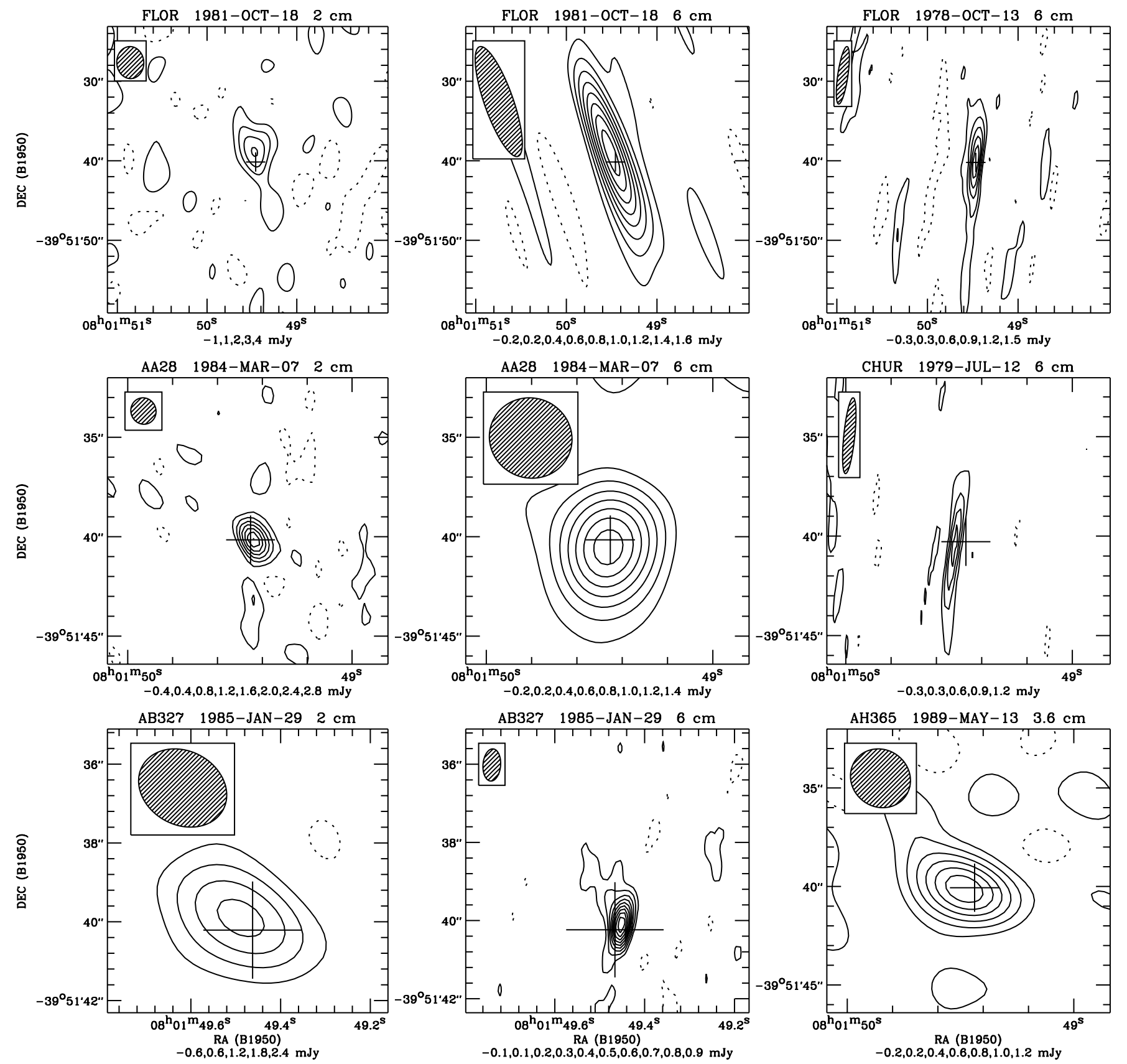

Fig. 5. Same as Fig. 4, but for the VLA archive data at 2, 3.6 and $6 \mathrm{~cm}$. Only those observations having a detection are shown. Note the different spatial scales and different contour levels of the maps.

measured peak intensity is systematically higher (by $\sim 40 \%$ ) than the flux derived from the Gaussian fit. This suggests that, due to phase problems, the source is not quite a point source. The measured peak intensity is $2 \mathrm{mJy}$. Minor comments on other observations are given in Table 5.

In general, the agreement of the fluxes we derived with published values is quite good (see notes to Table 5). Only for the AA28 $-6 \mathrm{~cm}$ observation do we find a value $(1.49 \pm 0.1 \mathrm{mJy})$ significantly higher than the published value $(1.3 \pm 0.1 \mathrm{mJy}$, Bieging et al. 1989).

There is an additional observation available (AC308 see Table 1) made at $20 \mathrm{~cm}$. This observation is part of the NRAO VLA Sky Survey (NVSS - Condon et al. 1998). We checked this survey and found that $\zeta$ Pup was not detected (but EQ 0801-398 is detected).

\subsection{Long term variability}

We now compare the different VLA observations, wavelength by wavelength, to see if we can detect variability at timescales much longer than the rotation period.

We have only two $2 \mathrm{~cm}$ flux determinations. The error bars do not overlap, which might suggest variability. However, we recall the considerable difficulties in the reduction of these data (Sect. 4.2), and based on that, we do not consider them to present evidence of variability. For completeness, we also 


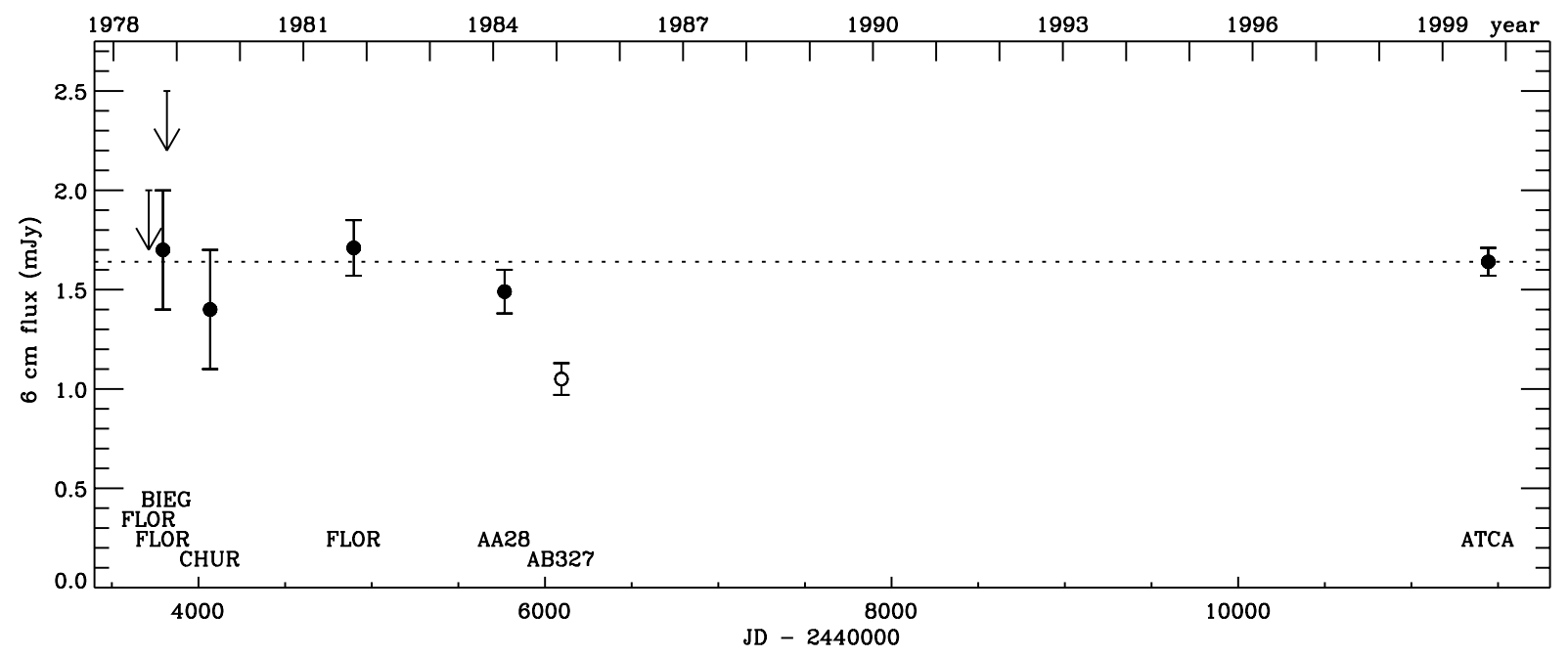

Fig. 6. The $6 \mathrm{~cm}$ fluxes of $\zeta$ Pup as a function of time. The open circle indicates an observation (AB327) that might be unreliable (see Sect. 4.2). The dotted line shows the flux from the ATCA combined data.

mention the $2 \mathrm{~cm}$ observations of $\zeta$ Pup by Morton \& Wright (1979): they list $7.2 \pm 1.1 \mathrm{mJy}$ as the average of two observing runs. This value is significantly higher than ours. However, these observations were made with a single-dish antenna (Parkes 64-m), which has a beam of 2!3. When targeting $\zeta$ Pup, the beam also covers S2 = EQ 0801-398 (see Fig. 1), which will dominate the measured flux. These data can therefore not be used to look for variability of $\zeta$ Pup.

The single VLA $3.6 \mathrm{~cm}$ observation $(1.4 \pm 0.3 \mathrm{mJy})$ can be compared to the ATCA determination $(2.38 \pm 0.09 \mathrm{mJy})$. In Sect. 4.2 we showed that the AH365 observation was quite problematic. Comparing the ATCA measurements of sources S2 = EQ 0801-398 and S3 = NVSS J080327-395828 to the AH365 measurements shows only a $20 \%$ effect for S2, and none for S3, suggesting that the Gaussian fit fluxes would be reliable. In that case, the AH365 $\zeta$ Pup flux would be significantly different from the ATCA determination. However AH365 shows such an accumulation of problems (the flux of the secondary calibrator is considerably less than expected, there are reasonably high phase changes and there is the difference between peak intensity and Gaussian integrated flux) that we do not consider this convincing evidence of variability.

The single $20 \mathrm{~cm}$ determination is compatible with the two upper limits.

The only wavelength for which we have a reasonable number of flux determinations, is $6 \mathrm{~cm}$. Figure 6 shows these fluxes as a function of time. The flux from the ATCA combined data goes through the error bars of almost all observations, indicating that there is no detectable variability.

The only exception is the significantly lower flux of AB327. In Sect. 4.2 we discussed the phase problems of this observation, which lead to the lower flux. Another possible factor might be that we are starting to resolve the stellar wind of $\zeta$ Pup (this is the observation with the highest spatial resolution we have). To estimate how much flux we would lose, we use a Wright \& Barlow (1975) approach. By their definition of the characteristic radius $\left(R_{v}\right)$ of the radio-emitting region, the observed flux $\left(S_{v}\right)$ is given by:

$S_{v}=10^{-26} \int_{R_{v}}^{+\infty} \mathrm{d} r \frac{4 \pi r^{2}}{D^{2}} K(v, T) \gamma\left(\frac{\dot{M}}{4 \pi r^{2} v_{\infty} \mu m_{\mathrm{H}}}\right)^{2} B_{v}(T)$,

where $r$ is the radius, $D$ the distance to the star, $K(v, T)$ the freefree absorption coefficient, $\gamma$ the ratio of electron to ion number densities, $\dot{M}$ the mass loss rate, $v_{\infty}$ the terminal velocity, $\mu$ the average atomic mass, $m_{\mathrm{H}}$ the proton mass and $B_{v}(T)$ the Planck function at frequency $v$ and temperature $T$. All units are cgs, except for $S_{v}$ which is in mJy. If, instead of integrating to $+\infty$, we only integrate to a radius $R_{\max }$, we lose a fraction of the flux given by:

$\frac{\Delta S_{v}}{S_{v}}=\frac{R_{v}}{R_{\max }}$.

The beamsize $\left(\theta_{\text {beam }}\right)$ determines $R_{\max }$ by: $\theta_{\text {beam }}=2 R_{\max } / D$. Combining this with the Wright \& Barlow expression for $R_{v}$, we get:

$\frac{\Delta S_{v}}{S_{v}}=3.8 \times 10^{5} \frac{\left(\gamma g Z^{2}\right)^{1 / 3} T^{-1 / 2}}{D \theta_{\text {beam }}}\left(\frac{\dot{M} \lambda}{\mu v_{\infty}}\right)^{2 / 3}$,

where $\dot{M}$ is in $M_{\odot} / \mathrm{yr}, v_{\infty}$ in $\mathrm{km} \mathrm{s}^{-1}, \lambda$ in cm, $\theta_{\text {beam }}$ in $\operatorname{arcsec}, D$ in kpc. We take $\dot{M}=5.9 \times 10^{-6}$ (Puls et al. 1996), $\mu=1.4$, $\gamma=1, Z=1$ and the Gaunt-factor $g=7$. Other parameters are taken from Table 2 . With a beam of $\theta_{\text {beam }}=0$.' 7 , we find a flux loss of $6-12 \%$, depending on whether we take a hot wind $(42000 \mathrm{~K})$ or a cool wind $(10000 \mathrm{~K})$. If we use the mass loss rate derived from our model for the radio observations (Table 7), we find a 4-8\% effect.

The low AB327 flux can thus not be explained by our starting to resolve the stellar wind, but is due to problems with the interpolation of the gain phases.

\section{JCMT observation}

We also determined the $850 \mu \mathrm{m}$ flux of $\zeta$ Pup using the Submillimetre Common-User Bolometer Array (SCUBA, 
Table 6. Submillimetre and radio fluxes of $\zeta$ Pup. Data from this paper, unless otherwise indicated.

\begin{tabular}{lll}
\hline \hline$\lambda$ & Flux (mJy) & Reference \\
\hline $850 \mu \mathrm{m}$ & $28 \pm 5$ & JCMT \\
$1.3 \mathrm{~mm}$ & $20.2 \pm 1.8$ & from Leitherer \& Robert (1991) \\
$2 \mathrm{~cm}$ & $4.3 \pm 0.9$ & VLA, FLOR 1981-10-18 \\
$2 \mathrm{~cm}$ & $2.9 \pm 0.3$ & Bieging et al. (1989), revised \\
$3.6 \mathrm{~cm}$ & $2.38 \pm 0.09$ & ATCA \\
$6 \mathrm{~cm}$ & $1.64 \pm 0.07$ & ATCA \\
$20 \mathrm{~cm}$ & $0.76 \pm 0.09$ & VLA \\
\hline
\end{tabular}

Holland et al. 1999) on JCMT (see Table 1). The instrument observes simultaneously at 450 and $850 \mu \mathrm{m}$ using two hexagonal arrays of bolometers. The sensitivity at $450 \mu \mathrm{m}$ is too low for a detection, so we will only discuss the $850 \mu$ m data. Our flexibly scheduled observations were taken in medium weather conditions $(850 \mu \mathrm{m}$ zenith opacity was $0.32-0.47)$ during a half-shift on 2000 October 12 . The beam at $850 \mu \mathrm{m}$ is $144^{\prime \prime} 5$.

As the data were collected in the same run as the $\epsilon$ Ori observation discussed by Blomme et al. (2002), we refer to that paper for details on the observation and the reduction. The total on-target integration time for $\zeta$ Pup was 15 min. After reduction, the rms on the $\zeta$ Pup observation turns out to be about $16 \%$. We tried variants in the reduction (e.g. using other bolometers than the inner ring for sky-noise removal), but this changes the flux by considerably less than $16 \%$.

The $\zeta$ Pup observations are preceded and succeeded by an observation of the calibrator $\mathrm{OH} 231.8$. Taking the average of these two calibration observations, we arrive at $31 \pm 5 \mathrm{mJy}$ for the flux. The error bar takes into account the measurement errors on the target and the calibrator as well as the calibration error. However, OH231.8 is somewhat variable and the flux of the calibrator at the time of our observation could be different from the reference flux we used.

If we use the HL Tau calibration observation that precedes our run, we have $28 \pm 5 \mathrm{mJy}$. Trying to use other calibrators that were observed during that night (but of course further away in time from our observation) tends to favour the lower value. In view of this we propose $28 \pm 5 \mathrm{mJy}$ as the best determination of the flux. The $\zeta$ Pup value is then also based on the same calibrator as the $\epsilon$ Ori observation analysed by Blomme et al. (2002).

If we want to compare our $850 \mu \mathrm{m}$ value with the $1.3 \mathrm{~mm}$ determination of Leitherer \& Robert (1991), we need to correct for the difference in wavelength. If we assume an $\alpha=$ 0.6 spectrum, we find that our value corresponds to $22 \pm 4 \mathrm{mJy}$ at $1.3 \mathrm{~mm}$, which agrees very well with the Leitherer \& Robert value of $20.2 \pm 1.8 \mathrm{mJy}$. Our value is also compatible with the Altenhoff et al. (1994) upper limit of $33 \mathrm{mJy}$ at $1.2 \mathrm{~mm}$.
Table 7. Comparison to other mass loss rate determinations of $\zeta$ Pup.

\begin{tabular}{lll}
\hline \hline Wavelength & $\dot{M}\left(10^{-6} M_{\odot} / \mathrm{yr}\right)$ & \multicolumn{1}{c}{ Reference } \\
\hline radio & $2.4_{-0.7}^{+1.0}$ & Lamers \& Leitherer (1993) \\
$\mathrm{H} \alpha$ & $3.5_{-1.2}^{+1.9}$ & Lamers \& Leitherer (1993) \\
& 5.9 & Puls et al. (1996) \\
radio & 3.5 & this paper \\
\hline
\end{tabular}

\section{Discussion}

\subsection{Smooth wind model}

Table 6 summarizes the submillimetre and radio fluxes we will discuss in this section. Other fluxes either agree with these, or possible disagreements have been explained in Sect. 4.2.

From the $1.3 \mathrm{~mm}$ and $6 \mathrm{~cm}$ fluxes we can derive the spectral index $\alpha$ (defined by $F_{v} \propto \lambda^{-\alpha}$ ). The measured value of $\alpha=0.66 \pm 0.03$ defines a power law that goes through the error bars of all observations, with the exception of the $2 \mathrm{~cm}$ fluxes (where it is intermediate between the two determinations). This spectrum is somewhat steeper than expected from the Wright $\&$ Barlow (1975) model (where $\alpha=0.6$ ), indicating some discrepancy between the millimetre and radio fluxes.

To better quantify this discrepancy, we also made a smooth wind model for $\zeta$ Pup in the same way as in Runacres \& Blomme (1996). The model solves the equations of radiative transfer and statistical equilibrium in a spherically symmetric stellar wind, containing only hydrogen and helium. The density in the wind is determined by solving the time-independent hydrodynamical equations (following Pauldrach et al. 1986). When fitting the model to the observations, the visual and near-infrared fluxes were used to determine the interstellar extinction and the radio fluxes to determine the mass loss rate. The far-infrared and millimetre fluxes are unconstrained and can therefore be used to see how well the smooth wind model fits the observations. Further details of the model are given in Runacres \& Blomme. A new version of their $\zeta$ Pup model was calculated that uses the parameters listed in Table 2.

In fitting the model, we fixed the mass loss rate from our ATCA $6 \mathrm{~cm}$ observation. We choose this observation because it has the smallest error bar. The best fit gives a mass loss rate of $\dot{M}=3.5 \pm 0.1 \times 10^{-6} M_{\odot} /$ yr. The error bar on $\dot{M}$ corresponds only to the error bar on the $6 \mathrm{~cm}$ flux, and does not include the more important errors due to stellar parameters or distance. E.g., the error in the Hipparcos distance converts to a $(-0.9$, $+1.5) \times 10^{-6} M_{\odot} / \mathrm{yr}$ error on $\dot{M}$.

Because it is such a well-studied star, a large number of mass loss rate determinations have been made for $\zeta$ Pup. In our comparisons we will only consider the more recent determinations (Table 7). Our radio mass loss rate of $3.5 \times$ $10^{-6} M_{\odot} / \mathrm{yr}$ is different from the $5.9 \times 10^{-6} M_{\odot} / \mathrm{yr}$ value by Puls et al. (1996) based on fitting the $\mathrm{H} \alpha$ line profile. This difference is significant, considering that we used the same stellar parameters as they did. We recall that Petrenz \& Puls (1996) already noted this discrepancy and tried to explain it by invoking an equatorial density enhancement, caused by the large rotational velocity of the star. Our results are in acceptable 


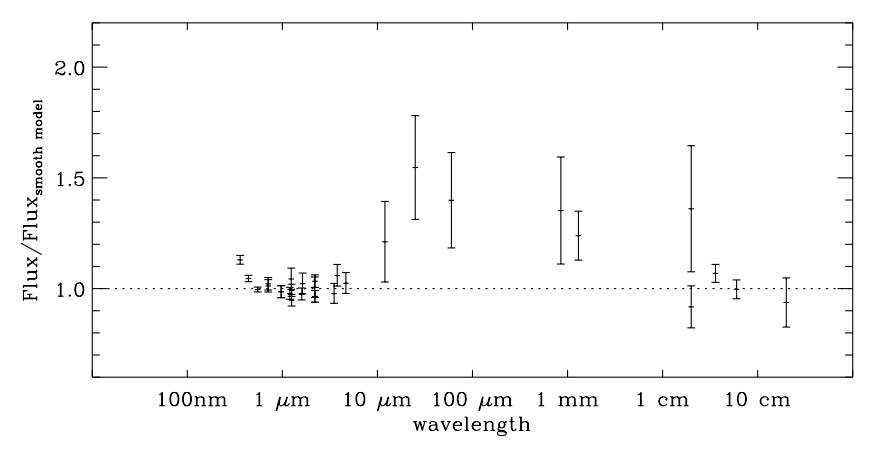

Fig. 7. Observed fluxes normalised to a smooth wind model. Observations above the dotted line point to additional emission that is not included in the smooth model.

agreement with both the radio and $\mathrm{H} \alpha$ determinations by Lamers \& Leitherer (1993), but we note that they used a simpler model for the $\mathrm{H} \alpha$ emission than Puls et al. Contrary to what the Lamers \& Leitherer numbers suggest, $\zeta$ Pup is therefore an exception to the usually good agreement between the $\mathrm{H} \alpha$ and radio mass loss rates.

The observed fluxes are compared to the best-fit smooth wind model in Fig. 7. The figure shows the millimetre and radio observations listed in Table 7, supplemented by visual and infrared data listed in Runacres \& Blomme (1996). As found by Runacres \& Blomme, there is an excess flux at millimetre wavelengths. Due to our somewhat higher $6 \mathrm{~cm}$ flux, the effect found here $(24 \%)$ is somewhat lower than what they found $(35 \%)$. The present result is significant at the 2 sigma level. In view of the large error bars, it is not clear what happens at $2 \mathrm{~cm}$, but the $3.6 \mathrm{~cm}$ flux could be marginally in excess. The $20 \mathrm{~cm}$ flux is in good agreement with the smooth wind model. We stress that the same discrepancy is found when a much simpler Wright \& Barlow (1975) model is used instead of our smooth wind model.

\subsection{Explaining the millimetre excess}

The millimetre excess is the most important result from this work. Various explanations will be considered in this section. To study the excess we will make considerable use of models similar to the one developed by Wright \& Barlow (1975).

We start by noting that the Wright \& Barlow formula for the flux is not very sensitive to temperature, so a radial gradient of the temperature will not explain the excess. There could be an indirect effect of the temperature however, due to the recombination of important ions. This explanation has been proposed by Leitherer \& Robert (1991). As $\mathrm{He}^{++}$will recombine much more quickly than $\mathrm{H}^{+}$, we only consider the recombination of $\mathrm{He}^{++}$. A careful evaluation of the ionizationdependent factors in the Wright \& Barlow (1975) formula for the radio flux shows that, if we assume all helium to be $\mathrm{He}^{++}$in the millimetre formation region and all helium to be $\mathrm{He}^{+}$in the centimetre formation region, the discrepancy between the fluxes can be explained. It should be noted that our own smooth-wind model does not show recombination, but it does not include important effects, such as line blanketing due to metals. Recent models of early-type stars arrive at lower effective temperatures than the one we used here (Bianchi \& Garcia 2002; Bianchi et al. 2003; Martins et al. 2002), which would favour recombination. Further evidence that recombination occurs, comes from models of the stellar wind of $\zeta$ Pup (Hillier et al. 1993; Pauldrach et al. 2001; Puls 2003, pers. comm.). Due to the recombination in these models, it is necessary to assume that the X-rays are formed out to large distances in the wind ( $>100 R_{*}$ ), because X-rays formed too close to the star get absorbed. However, the X-ray spectral lines of $\zeta$ Pup (Cassinelli et al. 2001; Kahn et al. 2001) seem to require formation closer to the stellar surface and less opacity in the wind (Kramer et al. 2003). This fact is difficult to reconcile with $\mathrm{He}$ recombination.

A slower velocity law has been claimed to explain the farinfrared discrepancies seen in Fig. 7 (Kudritzki \& Puls 2000). To explain the millimetre discrepancies would require a very much slower velocity law. To investigate this we adapted the Wright \& Barlow (1975) model to include a $\beta$-type velocity law. In such case, an analytical solution is no longer possible, and all integrations were done numerically. We find that $\beta \approx 5$ is needed to explain the millimetre fluxes, which is not compatible with other observational indicators such as the $\mathrm{H} \alpha$ profile, which requires $\beta \approx 1.15$ (Puls et al. 1996).

The existence of a flux excess at millimetre wavelengths can also be due to structure in the wind. As there is a wealth of evidence for structure in these winds, it seems obvious to explore a model based on that.

The model in question is the same as used in Blomme et al. (2002) for $\epsilon$ Ori. The smooth wind opacity in a Wright \& Barlow (1975) model is multiplied by the clumping factor to get the clumped wind opacity. The clumping factor is defined as $\left\langle\rho^{2}\right\rangle /\langle\rho\rangle^{2}$, where the symbol $<>$ stands for a time-average, which we have approximated by integrating over a small volume of the wind. Optical depth, emergent intensity and flux are then calculated as in Wright \& Barlow. As our model allows the clumping factor and velocity to change as a function of distance, all integrations have to be done numerically. We used a run of the clumping factor based on the work of Runacres \& Owocki (2001), who calculated time-dependent hydrodynamical models to study the effect of the line-driving instability at large distances from the star. The clumping factor in their models rises to reach a maximum rather far away from the star (10-50 $R_{*}$ ) and then decreases again. If we simplify their results somewhat, we can approximate the typical behaviour for the clumping factor by a piece-wise linear curve fixed by specifying three points. We let the clumping factor rise linearly from close to the surface of the star (point 1) to a certain distance (point 2), and then let it fall off again linearly till it becomes one (point 3 ). The inset to Fig. 8 shows an example (dotted line). We also used piece-wise linear curves fixed by four points. By setting the clumping factor equal to one at large distances, we ensure that there is no excess at radio wavelengths.

Figure 8 shows a number of fits we attempted. Although no unique solution is found, it is quite clear that the clumping factor has to be significantly higher than 1 in the inner part of the wind, and needs to diminish considerably beyond $70 R_{*}$. To stress this last point, Fig. 8 includes a model with substantial 


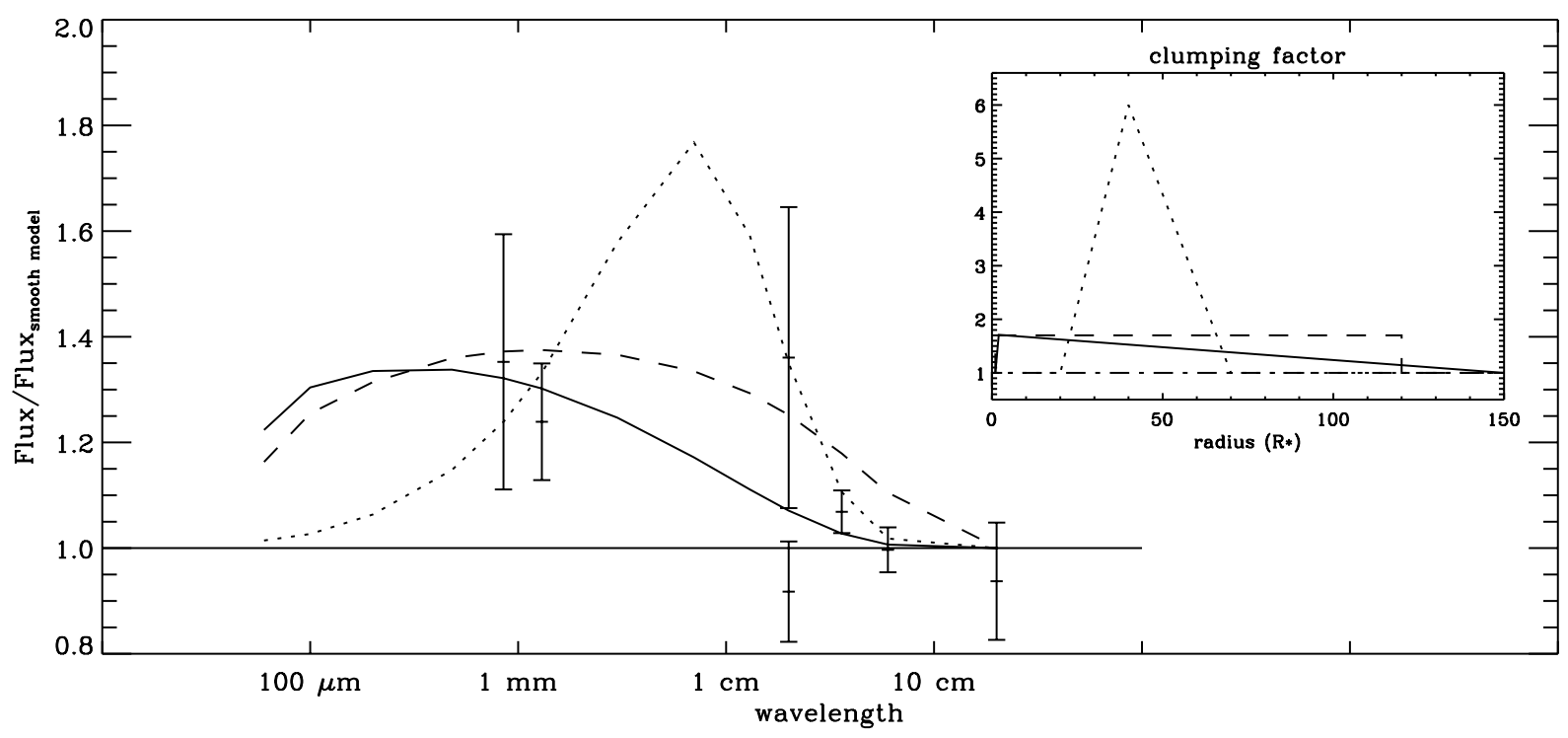

Fig. 8. The millimetre excess is fitted with a structured wind model. Three possible runs of the clumping factor as a function of radius are used (inset). The dashed line presents a model that has too much structure too far out in the wind to be able to explain the observations.

clumping up to $120 R_{*}$ (dashed line); the fluxes clearly overshoot the 3.6 and $6 \mathrm{~cm}$ observations. The fact that structure diminishes beyond a certain distance is similar to what we found for $\epsilon$ Ori (Blomme et al. 2002), except that for $\epsilon$ Ori, this started happening around $\sim 40 R_{*}$.

While structure diminishes around $70 R_{*}$, it does not necessarily disappear. For $\epsilon$ Ori we showed that a model where structure persists up to large distances (with a constant clumping factor) could equally well explain the observations, provided we reduce the mass loss rate accordingly. For $\zeta$ Pup, we see that the $20 \mathrm{~cm}$ observation is in reasonable agreement with the $6 \mathrm{~cm}$ one. This shows that, if there is still clumping left, it falls off considerably slower than it does in the inner $70 R_{*}$ of the wind. Obviously, a more accurate $20 \mathrm{~cm}$ flux and observations in the $1 \mathrm{~mm}-3.6 \mathrm{~cm}$ region will further constrain the extent and amount of structure.

The above model was very much inspired by the smallscale structure expected from the instability of the radiative driving mechanism. However, CIRs, a disk or a polar enhancement should have similar effects on the millimetre flux. In these cases the $70 R_{*}$ radius will be significant as well, in that it shows where the structure diminishes substantially, or maybe even disappears. A detailed comparison of models for different types of structure is beyond the scope of the present paper.

\section{Conclusions}

Radio observations of $\zeta$ Pup covering about two rotational periods, supplemented by archive observations covering a much longer time scale, do not show variability at more than the $\pm 20 \%$ level. The long integration time gives us an accurate flux determination of $2.38 \pm 0.09 \mathrm{mJy}$ at $3.6 \mathrm{~cm}$ and $1.64 \pm 0.07 \mathrm{mJy}$ at $6 \mathrm{~cm}$. These values are slightly higher than the ones previously known.
Converting the fluxes into a mass loss rate, we find $\dot{M}=$ $3.5 \times 10^{-6} M_{\odot} / \mathrm{yr}$. This value confirms the significant discrepancy with the $\mathrm{H} \alpha$ mass loss rate (Petrenz \& Puls 1996).

A smooth wind model shows that the millimetre fluxes are too high compared to the radio fluxes. While recombination of helium in the outer wind cannot be discounted as an explanation, we favour a model that ascribes the discrepancy to structure. A simple model shows a substantial decay, or maybe disappearance, of structure beyond $70 R_{*}$. Figure 8 shows how observations at wavelengths between $1 \mathrm{~mm}$ and $3.6 \mathrm{~cm}$ can further constrain models for structure.

The present data do not allow a distinction between the various types of structure (stochastic, CIRs, disk or polar enhancement). Attempting to detect variability at far-infrared or millimetre wavelengths should provide better constraints on the azimuthal symmetry of the structure, thereby allowing us to decide whether it is in the form of CIRs.

Acknowledgements. We thank J. Vandekerckhove for his help with the reduction of the VLA data. We are grateful to T. Lowe for making the JCMT observation. We also thank the original observers of the VLA archive data we used. We thank J. Puls for information about the helium recombination in the stellar wind models. This work benefitted from discussions with S. Owocki. This research has made use of the SIMBAD database, operated at CDS, Strasbourg, France and NASA's Astrophysics Data System Abstract Service. We also consulted the High Energy Astrophysics Science Archive Research Center (HEASARC), provided by NASA's Goddard Space Flight Center. M.C.R. acknowledges support from ESA-Prodex project no. 13346/98/NL/VJ(ic), financed by ESA-Prodex. Part of this research was carried out in the framework of the project IUAP P5/36 financed by the Belgian State, Federal Office for Scientific, Technical and Cultural Affairs.

\section{References}

Abbott, D. C., Bieging, J. H., Churchwell, E., \& Cassinelli, J. P. 1980, ApJ, 238, 196 
Altenhoff, W. J., Thum, C., \& Wendker, H. J. 1994, A\&A, 281, 161 Balona, L. A. 1992, MNRAS, 254, 404

Barker, P. K., Landstreet, J. D., Marlborough, J. M., Thompson, I., \& Maza, J. 1981, ApJ, 250, 300

Berghöfer, T. W., \& Schmitt, J. H. M. M. 1994, A\&A, 290, 435

Berghöfer, T. W., Baade, D., Schmitt, J. H. M. M., et al. 1996, A\&A, 306, 899

Bianchi, L., \& Garcia, M. 2002, ApJ, 581, 610

Bianchi, L., Garcia, M., \& Herald, J. 2003, Rev. Mex. Astron. Astrofis., Serie Conf., 15, 226

Bieging, J. H., Abbott, D. C., \& Churchwell, E. B. 1989, ApJ, 340, 518

Bjorkman, J. E., \& Cassinelli, J. P. 1993, ApJ, 409, 429

Blomme, R., Prinja, R. K., Runacres, M. C., \& Colley, S. 2002, A\&A, 382, 921

Bohannan, B., Abbott, D. C., Voels, S. A., \& Hummer, D. G. 1990, ApJ, 365, 729

Briggs, D. S. 1995, High Fidelity Deconvolution of Moderately Resolved Sources, Ph.D. Thesis, The New Mexico Institute of Mining and Technology, Socorro, New Mexico

Cassinelli, J. P., Miller, N. A., Waldron, W. L., MacFarlane, J. J., \& Cohen, D. H. 2001, ApJ, 554, L55

Chesneau, O., \& Moffat, A. F. J. 2002, PASP, 114, 612

Collura, A., Sciortino, S., Serio, S., et al. 1989, ApJ, 338, 296

Condon, J. J., Cotton, W. D., Greisen, E. W., et al. 1998, AJ, 115, 1693

Cranmer, S. R., \& Owocki, S. P. 1996, ApJ, 462, 469

Eversberg, T., Lépine, S., \& Moffat, A. F. J. 1998, ApJ, 494, 799

Feldmeier, A., Kudritzki, R.-P., Palsa, R., Pauldrach, A. W. A., \& Puls, J. 1997, A\&A, 320, 899

Fomalont, E. B., \& Perley, R. A. 1999, in Synthesis Imaging in Radio Astronomy II, ed. G. B. Taylor, C. L. Carilli, \& R. A. Perley, ASP Conf. Ser., 180, 79

Fullerton, A. W., Gies, D. R., \& Bolton, C. T. 1996, ApJS, 103, 475

Gontcharov, G. A., Andronova, A. A., Titov, O. A., \& Kornilov, E. V. 2001, A\&A, 365, 222

Hanbury Brown, R., Davis, J., \& Allen, L. R. 1974, MNRAS, 167, 121

Harries, T. J., \& Howarth, I. 1996, A\&A, 310, 533

Hendry, E. M., \& Bahng, J. D. R. 1981, JApA, 2, 141

Hillier, D. J., Kudritzki, R. P., Pauldrach, A. W. A., et al. 1993, A\&A, 276, 117

Holland, W. S., Robson, E. I., Gear, W. K., et al. 1999, MNRAS, 303, 659

Howarth, I. D., \& Brown, A. B. 1991, in Wolf-Rayet Stars and Interrelations with Other Massive Stars in Galaxies, ed. K. A. van der Hucht, \& B. Hidayat (Dordrecht: Kluwer), IAU Symp., 143,315

Howarth, I. D., Prinja, R. K., \& Massa, D. 1995, ApJ, 452, L65

Jones, P. A. 1985, MNRAS, 216, 613

Kahn, S. M., Leutenegger, M. A., Cottam, J., et al. 2001, A\&A, 365, L312
Kramer, R. H., Cohen, D. H., \& Owocki, S. P. 2003, ApJ, 592, 532

Kudritzki, R. P., \& Puls, J. 2000, ARA\&A, 38, 613

Kudritzki, R. P., Simon, K. P., \& Hamann, W.-R. 1983, A\&A, 118, 245

Lamers, H. J. G. L. M., \& Leitherer, C. 1993, ApJ, 412, 771

Leitherer, C., \& Robert, C. 1991, ApJ, 377, 629

Long, K. S., \& White, R. L. 1980, ApJ, 239, L65

Lucy, L. B. 1982a, ApJ, 255, 286

Lucy, L. B. 1982b, ApJ, 255, 278

Marchenko, S. V., Moffat, A. F. J., Van der Hucht, K. A., et al. 1998, A\&A, 331, 1022

Martins, F., Schaerer, D., \& Hillier, D. J. 2002, A\&A, 382, 999

Massa, D., Fullerton, A. W., Nichols, J. S., et al. 1995, ApJ, 452, L53

Mermilliod, J.-C. 1987, A\&AS, 71, 413

Moffat, A. F. J., \& Michaud, G. 1981, ApJ, 251, 133

Morton, D. C., \& Wright, A. E. 1979, in Mass loss and evolution of O-type stars, ed. P. S. Conti, \& C. W. H. de Loore (Dordrecht: Kluwer), IAU Symp., 83, 155

Mullan, D. J. 1986, A\&A, 165, 157

Oskinova, L. M., Clarke, D., \& Pollock, A. M. T. 2001, A\&A, 378, L21

Owocki, S. P. 2000, Radiatively Driven Stellar Winds from Hot Stars, in Encyclopedia of Astronomy and Astrophysics, http://www.ency-astro.com (London: Nature Publishing Group, and Bristol: Institute of Physics Publishing)

Owocki, S. P., Cranmer, S. R., \& Gayley, K. G. 1996, ApJ, 472, L115

Owocki, S. P., Cranmer, S. R., \& Gayley, K. G. 1998, Ap\&SS, 260, 149

Pauldrach, A., Puls, J., \& Kudritzki, R. P. 1986, A\&A, 164, 86

Pauldrach, A. W. A., Kudritzki, R. P., Puls, J., Butler, K., \& Hunsinger, J. 1994, A\&A, 283, 525

Pauldrach, A. W. A., Hoffmann, T. L., \& Lennon, M. 2001, A\&A, 375, 161

Petrenz, P., \& Puls, J. 1996, A\&A, 312, 195

Petrenz, P., \& Puls, J. 2000, A\&A, 358, 956

Perley, R. A., \& Taylor, G. B. 2002, The VLA Calibrator Manual http://www. aoc.nrao.edu/ gtaylor/calib.html

Prinja, R. K., Barlow, M. J., \& Howarth, I. D. 1990, ApJ, 361, 607

Prinja, R. K., Balona, L. A., Bolton, C. T., et al. 1992, ApJ, 390, 266

Puls, J., Kudritzki, R. P., Herrero, A., et al. 1996, A\&A, 305, 171

Reid, A. H. N., \& Howarth, I. D. 1996, A\&A, 311, 616

Runacres, M. C., \& Blomme, R. 1996, A\&A, 309, 544

Runacres, M. C., \& Owocki, S. P. 2001, A\&A, 381, 1015

Sault, B., \& Killeen, N. 1999, Miriad Users Guide http://www . atnf. csiro.au/computing/software/miriad

Sciortino, S., Vaiana, G. S., Harnden, F. R. Jr., et al. 1990, ApJ, 361, 621

Snow, T. P. Jr., York, D. G., \& Welty, D. E. 1977, AJ, 82, 113

Walborn, N. R. 1972, AJ, 77, 312

Wendker, H. J. 1995, A\&AS, 109, 177

Wright, A. E., \& Barlow, M. J. 1975, MNRAS, 170, 41 